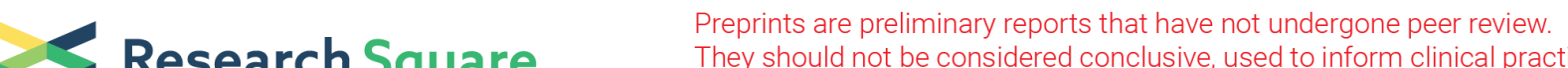 $\begin{array}{ll}\text { Research Square } & \text { They should not be considered conclusive, used to inform clinical practice, } \\ \text { or referenced by the media as validated information. }\end{array}$
}

\section{P2X7 Receptor of Microglia Mediates Neuropathic pain by Regulating Autophagy After Chronic- Constriction Injury}

\section{Qi Zhong}

Wuhan University Zhongnan Hospital

\section{Yifei Huang}

Wuhan University Zhongnan Hospital

\section{Bo Zhang}

Tongji Medical College of Huazhong University of Science and Technology: Huazhong University of Science and Technology Tongji Medical College

\section{Tingting Li}

Wuhan University Zhongnan Hospital

\section{Peng Fang}

Wuhan University Zhongnan Hospital

Jing Chang

Wuhan University Zhongnan Hospital

Chang chen

Wuhan University Zhongnan Hospital

Zongze Zhang ( $\boldsymbol{D}$ zhangzz@whu.edu.cn )

Wuhan University Zhongnan Hospital

\section{Research}

Keywords: P2X7R, Neuropathic pain, Microglia, Autophagy, Apoptosis

Posted Date: June 30th, 2021

DOI: https://doi.org/10.21203/rs.3.rs-663805/v1

License: (c) (i) This work is licensed under a Creative Commons Attribution 4.0 International License. Read Full License 


\section{Abstract}

P2X7 receptor is a crucial receptor related to neuronal activation, neurosensitization, and pain transmission, and increasing evidences indicated that glial cells is thought to be a major contributor to the chronic neuropathic pain after nerve injury. In present study, we designed to investigate whether the P2X7R in glial plays a role in chronic neuropathic pain. We divided adult male Sprague Dawley rats were respectively into four groups:(1) vehicle group (Veh), (2) CCI (C group), (3) P2X7 inhibitor group (P group), (4) CCl+ P2X7R inhibition (CP group). Behavior test, real-time polymerase chain reaction, western blot, immunofluorescence staining, and transmission electron microscope were used to analyze the scientific hypothesis. The results of the experiment is that(i) P2X7R of microglia was downregulated by A-70003 after CCI. (ii) Downregulation of P2X7R on microglia is coincident with remission of NP after CCI. (iii) P2X7R of microglia participates in NP via regulating autophagy and apoptosis. In summary, our results support P2X7R inhibition can counteract the CCl-induced NP due to microglia activation via a modulation of autophagy and apoptosis in $\mathrm{MPFC}$ and spinal cord. This may provide an importantly neuroprotective mechanism for the improved NP and also help devising new therapeutic to improve chronic pain in patients.

\section{Introduction}

Neuropathic pain (NP) can develop after lesion or disease in the sensory system, which serves no protective function and affects $7 \% \sim 8 \%$ of the population [1].Despite recent advances in the knowledge of nociceptive information processing in NP, which remains a vital major clinical and social problem [2], so it is still necessary to go further into the study of the pathophysiology of this condition to facilitate the development of new more effective drugs or, at least, to repurpose some that are currently in clinical use.

Recently, investigations have highlighted the role of glial cells in the development of chronic pain. Particularly, researches demonstrated that peripheral nerve injury can result in microglia and astrocyte activation of medial prefrontal cortex (MPFC) area in NP models [3, 4]. Activated microglia release various algesic substances that enhance pain transmission by neurons. Autophagy, as a process to remove and recycle aggregated proteins, is an important function for maintaining cellular homeostasis, regulated by the activation of astrocytes and has been participated in allodynia and hyperalgesia. Piao et al [5] reported that lack of the p62 autophagic protein plays a key role in the pathophysiology of NP. Autophagy inducers have been shown to protect neurons, findings indicated that NP activates autophagy [6], and increased glial autophagy activity exerted a protective effect in NP via inflammasome inactivation [7]. However, how glial autophagy mediated NP has not been fully investigated.

In addition, P2X7 receptors are widely distributed in the central nervous system neurons, such as microglia and astrocytes. They are closely associated with pathologic pain, such as in the central nervous system, where they are expressed primarily in microglia cells and are thought to perform similar functions by inducing the release of inflammatory cytokines [8]. Early reports of the use of antagonists and knockout mice suggest that the microglia P2X7R is involved in chronic neuropathic and inflammatory 
pain by releasing interleukin-1 $\beta(\mathrm{IL}-1 \beta)[9]$. Previous studies have shown that $\mathrm{P} 2 \mathrm{X} 7 \mathrm{R}$ is upregulated in the spinal cord after nerve injury [10]. In addition, P2X7R seems to play an important role in NP [11]. For example, P2X7R-knockout mice fail to develop behavioral hypersensitivity after partial sciatic nerve ligation. Moreover, studies have shown that an autophagy inducer could result in long-lasting analgesia, inhibit IL-1 $\beta$ secretion [12], improves nerve myelination [13]and prevent pain chronification [14]. Takenouchi et al [15] proposed a mechanism for the P2X7R signaling pathway that may play an important role in activated microglial cells. However, whether P2X7R in glial cells contribute to NP through autophagy is unknown.

Therefore, the research hypothesis is that P2X7R in glials plays a role in chronic pain, and its descending pathway may be associated with autophagy. In the study, we established a chronic pain model for rats, and applied P2X7R inhibitor. After that, mPFC and spinal cord regions were selected and the relevant molecular biological level was studied to observe the indicators and demonstrate the hypothesis.

\section{Materials And Methods}

\section{Animal Studies}

The ethical approval for this experiment was approved by the Ethical Committee of the Animal Use and Care Committee of Zhongnan Hospital, and all animal experiments complied with the ARRIVE guidelines and were carried out in accordance with the Guide for the Care and Use of Laboratory Animals.

\section{Animals}

Five-Eight-week-old adult male Sprague Dawley rats wild-type mice with weight ranging from 200 to $300 \mathrm{~g}$ were obtained from the experimental animal center of Wuhan University School of Medicine. Prior to the study, animals were housed and fed at a temperature of $23 \pm 2^{\circ} \mathrm{C}$ and a humidity of $60-70 \%$, with a $12-\mathrm{h}$ light/dark cycle and free access to food and water. All experiments were conducted between 9 am and 5 pm. Mice were positioned on a heating pad to prevent hypothermia during experiments.

\section{Chronic-Constriction Injury Model}

The Chronic-Constriction Injury (CCl) model was established as previously described (Bennett and Xie, 1988). Briefly, the rats were anesthetized with pentobarbital sodium (40 mg/kg, i.p.), an incision was made on the lateral surface of the midthigh, the biceps femoris muscle was blunt-dissected, and the left sciatic nerve was exposed and loosely ligated with $4-0$ silk at four sites with an interval of $1 \mathrm{~mm}$. For the sham group, all procedures were identical to those in the $\mathrm{CCl}$ group, except that there was no nerve ligation. Afterward, the muscle and skin incisions were closed separately. The wound was sutured with 3 -0 silk.

P2X7R inhibition (A-740003 [N-(1-\{ [(Cyanoimino) (5-quinolinylamino) methyl]amino\}-2,2dimethylpropyl)-2-(3,4-dimethoxyphenyl) acetamide], a selectively P2X7 antagonist, Tocris, China) was injected to mPFC (bregma rostrocaudal $3.2 \mathrm{~mm}$, mediolateral $0.7 \mathrm{~mm}$, dorsoventral $3.5 \mathrm{~mm}$ ) of rat by 
bilateral stereotaxic (Techman, DW-2000, Chengdu, China). The rats were randomly divided into four groups according to the random number table: (1) vehicle group (Veh), which received vehicle (PBS, 0.5 $\mu$ l) (Aspen, AS1025, Wuhan, China) in the bilateral mPFC of the rats; (2) CCl group(C group), which was established CCI model; (3) P2X7 inhibitor group (P group), which received A-740003 (100M) in the bilateral mPFC of the rats at the 14th day for three consecutive days; (4) CCl + P2X7R inhibition (CP group), which received $\mathrm{A}-740003(100 \mathrm{M})$ in the bilateral mPFC of the rats at the 14 th day of $\mathrm{CCl}$ for three consecutive days. A-740003 was freshly dissolved in DMSO.

\section{Behavioral test}

Pain-related behavioral tests were performed according to previous described methods. All animals were acclimated to the testing environment two days before baseline testing. Von Frey hairs were used to stimulate the plantar surface of the hind paw to evaluate paw-withdrawal threshold (PWT), and 50\% PWT was determined using the up-down method. Briefly, a series of Von Frey hairs with ascending stiffness was used to stimulate the plantar surface of the hind paw, a positive response was recorded if the paw was suddenly withdrawn upon application of a filament as well as licking or biting of stimulated paw. Testing of a filament was done for five rounds per paw. The threshold filament size was set as the smaller filament resulted in three withdrawals out of five successive trials. The paw-withdrawal latency (PWL) was measured by the plantar test (7370, Ugo Basile, Comeria, Italy) and used the method of Hargreaves et al. Briefly, a radiant heat source beneath a glass floor was applied at the plantar surface of the hind paw. Each rat was tested three times with 5 min intervals between consecutive, alternating tests. The average of the three measurements was taken as the result per test.

\section{RT-PCR for P2X7R mRNA}

The area of MPFC in brain and spinal cord were collected and extracted using TRIzol reagent (Invitrogen ${ }^{\mathrm{T}}$, USA).The concentration of RNA was detected using ultraviolet absorption spectrometry. The cDNA was synthesized using EntiLink ${ }^{\text {Tm }} 1$ st Strand cDNA Synthesis Kit(ELK Biotechnology,EQ003). For quantitative real-time PCR reaction, EnTurbo ${ }^{\text {TM }}$ SYBR Green PCR SuperMix[ELK Biotechnology,EQ001]was used in StepOne ${ }^{\mathrm{TM}}$ Real-Time PCR detection system (Life Technologies, USA). The PCR was carried our at the following condition: pre-denaturing at $95^{\circ} \mathrm{C}$ for $3 \mathrm{mins}$, followed by 40 cycles of annealing reaction at $58^{\circ} \mathrm{C}$ for $30 \mathrm{~s}$, and extension $72^{\circ} \mathrm{C}$ for 30 s. The PCR primers for P2X7R are as follows: forward $5^{\prime}-$ GAAAAGCGGACATTGATCAAAG-3'and reverse5'- CAAGTCAATACACACGGTGGC', respectively, and the product size was 147 bp. $\beta$-actin was used as references for mRNAs. Each sample was analyzed in triplicate.

\section{Western blot analysis}

Animals were anesthetized after behavioral tests at predefined time points, the lumbar spinal cords and the area of $\mathrm{mPFC}$ in brain were removed, and the collected tissues were frozen in liquid nitrogen and stored at $-80^{\circ} \mathrm{C}$. Tissue samples were homogenized in RIPA buffer containing protease and phosphatase inhibitor. Lysates were centrifuged at $12000 \mathrm{rpm}$ for $15 \mathrm{~min}$ at $4^{\circ} \mathrm{C}$, and protein concentration was determined by a bicinchoninic acid (BCA) Protein Assay Kit (Beyotime Institute of 
Biotechnology, Shanghai, China).Samples with equal amount of protein were separated by $9 \%-12 \%$ PAGE (40g total protein per lane) and transferred onto polyvinylidene difluoride membranes (Millipore, USA), followed by blockage with $5 \%$ skim milk for $2 \mathrm{~h}$ and incubation overnight with the following primary polyclonal antibodies: rabbit anti-P2X7R (1:250; Alomone, Israel),rabbit anti-LC3B (1:1000, Cell Signaling Technology, Beverly, MA, United States), rabbit polyclonal anti Beclin 1 (1:1000, MBL International Corporation, Nagoya, Japan), rabbit anti-Bcl-2 (1:1000; 2876, Cell Signaling Technology, USA), rabbit antiBax (1:1000; 2772, Cell Signaling Technology, USA), rabbit anti-cleaved-Caspase-3 (1: 1000; 9661, Cell Signaling Technology, USA) .The membranes were washed and then incubated with secondary antibodies (anti-rabbit IgG at 1: 10000, Aspen, USA). The immune complexes were identified using an enhanced chemiluminescence (ECL) detection system. $\beta$-actin (1:10000, Tdy Biotech, Beijing, China) and GAPDH (1:200; Santa Cruz Biotechnology) was blotted on the same membrance as a loading control.

\section{Transmission electron microscopy study}

After the brain and spinal cord tissues were fixed with paraformaldehyde, and removed, then fixation in $2.5 \%(\mathrm{w} / \mathrm{v})$ glutaraldehyde overnight, the specimens were post-fixed in $2 \%(\mathrm{v} / \mathrm{v})$ osmium tetroxide and blocked with $2 \%(\mathrm{v} / \mathrm{v})$ uranyl acetate. Tissues were embedded in araldite after dehydration in a series of acetone washes. Semi-thin sections were cut and toluidine blue staining was performed for observation of localization. Finally, ultra-thin sections from at least three blocks per sample were cut and observed using a transmission electron microscopy (TEM)

\section{Immunostaining and image analysis}

Four mice in each group were humanely sacrificed at $1 \mathrm{~h}$ after the A740003 injection at the last time. The rats were deeply anaesthetized and transcardially perfused with $0.9 \%$ ice-cold saline solution followed by $4 \%$ paraformaldehyde(Aspen, Wuhan, China) at $4^{\circ} \mathrm{C}$. The fixed brain and spinal cord were immersed in the same fixative overnight, and embedded in paraffin. The paraffin-embedded tissue arrays were performed in sections for immuostaining .For staining P2X7R-positive cells, sections of Spinal cord slice were incubated with primary antibody (rabbit anti-P2X7R, 1:50, APR008, Alomone, Israel) and then biotinconjugated secondary antibody (goat anti-rabbit IgG, 1: 50, Aspen, USA).For P2X7R-Iba1 (ionized calcium binding adaptor molecule 1) / P2X7R-GFAP (glial acidic fibrillary protein) co-labeling in brain, sections were incubated with a mix of primary antibodies(rabbit anti-P2X7R,1:100,and mouse anti-lba1 or mouse anti-GFAP,1:100, Abcam, UK) and then secondary antibodies (goat anti-rabbit IgG at 1:100, goat antimouse IgG at 1:100, Aspen, USA, DAPI). For double immunofluorescence staining, the primary antibody for P62 (rabbit anti-P62, 1:200, Proteintech, United States) was incubated together with the mouse monoclonal antibody (anti Iba1 or anti GFAP, 1:100, Abcam, UK) and then incubated with secondary antibodies (goat anti-rabbit IgG at 1:100, goat anti-mouse IgG at 1:100, Aspen, USA).Images were acquired using a fluorescence microscope (Olympus, Tokyo, Japan) equipped with an imaging system.

\section{Statistical analysis}

GraphPad Prism version 8 software was used for statistical analysis. Because of the available number of animals and estimated statistical power, the sample size was 6/group for Behavioral test, 4/group for 
PCR, western blotting, TEM and immunofluorescence analysis. Data were expressed as the mean \pm standard error of the mean (SEM). All analyses were conducted using SPSS 20 statistical software. More than two groups were compared, statistical evaluation of the data was performed with one-way analysis of variance (ANOVA) followed by Dunnett's post-hoc test. $P<0.05$ was considered to be significant for all statistical comparison.

\section{Results}

Inhibition of P2X7R can increase thermal and mechanical pain thresholds in rats with chronic neuropathic pain

NP seriously affects the health and life quality of patients. However, the mechanism of NP has not been fully investigated and there is still lack of effective treatment for it. Previous study showed that P2X7 was involved in the pain transmission and the occurrence of NP in mice, in this study, we planned to explore whether P2X7R played a role in the process of chronic pain.

As shown in Fig. 1A, rats established by $\mathrm{CCl}$ model showed significantly lower pain threshold than the control rats from the third day after surgery, and there was no significant difference in heat and mechanical perception of rats given P2X7R inhibitor alone compared with the control group (thermal pain thresholds: Day 3:13.20 \pm 1.67 for group $V, 13.55 \pm 2.90$ for group $P, 8.23 \pm 1.44$ for group $C, p<0.001$, $7.48 \pm 1.22$ for group CP, $p<0.001$; mechanical pain thresholds: Day 3:33.27 \pm 4.22 for group V, $35.64 \pm$ 4.76 for group $P$ and $19.89 \pm 5.46$ for group $C, p<0.001,21.79 \pm 2.47$ for CP group, $p<0.001$ ) and continued until 14 days after surgery (thermal pain thresholds: Day 7:14.32 \pm 0.97 for group V, $15.48 \pm$ 1.38 for group $P, 5.87 \pm 1.58$ for group $C, P<0.001,6.56 \pm 0.18$ for $C P$ group, $p<0.001$; Day $10: 15.24 \pm 1.28$ for group $V, 14.74 \pm 2.53$ for group $P, 5.76 \pm 1.00$ for group $C, p<0.001,4.62 \pm 1.37$ for $C P$ group, $p<$ 0.001 ; Day 14: $13.71 \pm 0.69$ for group $V, 13.99 \pm 1.11$ for group $P, 3.84 \pm 0.88$ for group $C, P<0.001,7.44 \pm$ 1.29 for group $C P, p<0.001$; Day $15: 14.23 \pm 1.37$ for group $V, 14.33 \pm 2.28$ for group $P, 3.60 \pm 0.85$ for group $C, P<0.001,7.10 \pm 1.10$ for group $C P, P=0.0010 D$ ay $16: 14.14 \pm 1.60$ for group $V, 14.52 \pm 0.42$ for group $P, 3.79 \pm 0.42$ for group $C, p<0.001,7.24 \pm 0.77$ for group $C P, p<0.001$; mechanical pain thresholds: Day 7: $37.71 \pm 2.52$ for $V$ group, $34.56 \pm 4.75$ for $P$ group, $15.65 \pm 6.19$ for $C$ group, $p=0.001,16.97 \pm 1.05$ for CP group, $p<0.001$; Day 10:36.57 \pm 1.50 for group $V, 35.89 \pm 3.64$ for group $P, 12.46 \pm 2.03$ for group $C$, $p<0.001,11.75 \pm 1.05$ for CP group, $p<0.001$; Day14:34.59 \pm 2.58 for group $V, 36.41 \pm 6.37$ for group $P$, $10.37 \pm 3.36$ for group $C, p<0.001,16.00 \pm 1.42$ for CP group, $p<0.001 \otimes D$ ay $15: 35.07 \pm 3.00$ for group $V$, $36.12 \pm 5.82$ for group $\mathrm{P}, 9.88 \pm 1.38$ for group $\mathrm{C}, \mathrm{P}<0.001,15.85 \pm 2.36$ for $\mathrm{CP}$ group, $p=$ $0.001 \otimes$ Day $16: 34.54 \pm 2.33$ for group $V, 34.53 \pm 3.15$ for group $P, 10.76 \pm 2.88$ for group $C, p<0.001,15.95$ \pm 1.62 for CP group, $p<0.001$ ). However, after treated with P2X7R inhibitor at the 14 th days of $\mathrm{CCl}, \mathrm{CP}$ group postoperatively suffered longer heat pain radiation than $\mathrm{C}$ group $(p<0.001)$, which verified that P2X7R can increase thermal and mechanical pain thresholds in chronic pain model rats.

\section{Chronic pain was partially dependent on P2X7R in MPFC and spinal cord}


Previous studies have shown that the MPFC, which integrates the sensory, emotional and attentional components of pain perception, is the focus of chronic pain. To explore whether P2X7R in the mPFC region is associated with chronic pain processes, we examined P2X7R levels using WB and PCR. As shown in Fig. 2, the content of P2X7R in the brain of chronic pain rats was significantly higher than that of control rats $(0.04 \pm 0.01$ for group $V, 0.04 \pm 0.00$ for group $P, 0.19 \pm 0.03$ for group $C, p<0.001,0.10 \pm$ 0.01 for group $C P, p<0.001$, Fig. $2 B$ ), and in the chronic pain group, $P 2 X 7 R$ inhibitor treatment reduced the content of P2X7R in CP group (group C vs. CP, $p<0.001$, Fig. 2B). PCR showed the same trend $(0.93 \pm$ 0.34 for group $V, 0.77 \pm 0.21$ for group $P, 4.89 \pm 0.48$ for group $C, P<0.001,2.88 \pm 0.71$ for $C P$ group, $p<$ 0.001 , group C VS CP, $p<0.001$, Fig. 2 C).

In addition, the spinal cord acts as the primary center of pain transmission and plays a role in chronic pain. As shown in Fig. 2, the trend of P2X7R content in the chronic pain spinal cord was consistent to that in the brain (western blot: $0.25 \pm 0.02$ for group $V, 0.27 \pm 0.03$ for group $P, 0.47 \pm 0.07$ for group $C, p<0.001$, $0.35 \pm 0.01$ for CP group, $p<0.001$, Group C VS CP, $P=0.012$, Fig. 2E; PCR: $0.90 \pm 0.55$ for $V$ group, $1.00 \pm$ 0.31 for $P$ group, $3.85 \pm 0.23$ for $C$ group, $<0.001,1.93 \pm 0.47$ for $C P$ group, $p<0.001$, group $C$ VS $C P, p<$ 0.001 , Fig. 2F).

\section{P2X7 elimination decreases autophagy and apotosis in rats of chronic pain}

Autophagy plays a key role in the progression of pain pathology, researches have demonstrated that the inhibition of autophagy can alleviate neuropathic pain behavior in animals. However, whether autophagy was regulated by P2X7R in chronic pain has not been fully investigated.

In Fig. 3A, the number of autophagosomes in chronic pain rats can be observed was significantly higher than that in control rats (mPFC: $7.97 \pm 1.94$ for group $V, 8.79 \pm 0.72$ for $P, 22.14 \pm 3.01$ for group $C, p<$ $0.001,14.03 \pm 1.62$ for CP group, $p<0.001$, Fig. $3 A$; spinal cord: $3.59 \pm 1.17$ for group $V, 3.97 \pm 1.35$ for group $\mathrm{P}, 16.93 \pm 4.15$ for group $\mathrm{C}, p<0.001,9.74 \pm 2.40$ for $\mathrm{CP}$ group, $p<0.001$, Fig. $4 \mathrm{~A}$ ), and in rats of the chronic pain group, treatment with $\mathrm{P} 2 \mathrm{X} 7 \mathrm{R}$ inhibitors reduced the number of autophagosomes in the model rats (mPFC: Group C vs. CP, $p=0.001$, Fig. 3B; spinal cord: Group C vs. CP, $p=0.017$, Fig. 4B). Moreover, we measured the levels of Beclin 1, LC3区 / LC3囚 in the mPFC and spinal cord at 14 days of model establishment (Fig. 3C,4C) using Western Blot. It was consistent with the trend of the number of autophagosomes (mPFC: Beclin 1: $0.25 \pm 0.05$ for $V$ group, $0.27 \pm 0.03$ for $P$ group, $0.82 \pm 0.08$ for $C$ group, $p<0.001,0.67 \pm 0.04$ for CP group, $p<0.001$, group $C$ vs. CP, $\mathrm{P}<0.001$, Fig. 3D; LC3 $\varangle /$ LC3区: $0.14 \pm$ 0.08 for $V$ group, $0.20 \pm 0.02$ for $P$ group, $0.42 \pm 0.03$ for $C$ group, $p<0.001,0.40 \pm 0.05$ for group $C P, p<$ $0.001, C$ vs. CP group, $P=0.018$, Fig. $3 E$; spinal cord: Beclin 1: $0.32 \pm 0.05$ for group $V, 0.25 \pm 0.07$ for group $P, 0.74 \pm 0.10$ for group $C, p<0.001,0.48 \pm 0.02$ for group $C P, p=0.001$, Group $C$ vs. CP group, $p=0.002$, Fig. 4D; LC3 $₫ /$ LC3 $₫: 0.16 \pm 0.04$ for $V$ group, $0.15 \pm 0.03$ for group $P, 0.45 \pm 0.02$ for group $C, p<0.001$, $0.26 \pm 0.01$ for group $C P, p<0.001$, group $C$ vs. $C P, p<0.001$, Fig. $4 \mathrm{E})$. It confirms that inhibition of P2X7R can reduce autophagy and apoptosis in chronic pain. 
Next, we measured the levels of apoptotic indexes Bcl-2, Bax, and Cleaved caspase 3 at 14 days after model establishment using Western Blot (Fig. 3F) in mPFC and spinal cord(Fig. 4F). It could be seen that the ratio of $\mathrm{Bax} / \mathrm{Bcl}-2$ in $\mathrm{mPFC}$ and spinal cord of chronic pain rats were significantly higher than those in the control group (mPFC: $0.27 \pm 0.17$ for group $V, 6.72 \pm 1.21$ for group $P, 0.23 \pm 0.02$ for group $C, p<0.001$, $1.92 \pm 0.63$ for the CP group, $p=0.001$, Fig. $3 \mathrm{G}$; spinal cord: $0.39 \pm 0.07$ for group $V, 7.00 \pm 1.60$ for group $P$, $0.38 \pm 0.10$ for group C, $p<0.001,1.38 \pm 0.34$ for CP group, $p<0.001$, Fig. $4 \mathrm{G}$ ), and Bax/Bcl-2 level decreased after treatment with P2X7R inhibitors (mPFC: Group C vs. CP group P, $p<0.001$, Fig. 3G; spinal cord: group C vs. CP, $p<0.001$, Fig. $4 \mathrm{G}$ ), the contents of Cleaved caspase 3 were consistent to the trend (mPFC: Cleaved caspase3:0.14 \pm 0.09 for $V$ group, $0.14 \pm 0.02$ for $P$ group, $0.57 \pm 0.07$ for $C$ group, $p<$ $0.001,0.36 \pm 0.07$ for CP group, $p<0.001$, group C vs. CP, $p<0.001$, Fig. $3 \mathrm{H}$; spinal cord: Cleaved caspase3:0.13 \pm 0.04 for $V$ group, $0.13 \pm 0.02$ for $P$ group, $0.59 \pm 0.07$ for $C$ group, $p<0.001,0.29 \pm 0.04$ for CP group, $p<0.001$, group C vs. CP, $p<0.001$, Fig. $4 \mathrm{H}$ ).

\section{P2X7R in microglia of the MPFC and spinal cord contributed to chronic pain but not in astrocytes}

In order to explore whether P2X7R in microglia plays important roles in chronic pain,double P2X7R and microglial immunostaining of $\mathrm{mPFC}$ and spinal cord were performed 14 days after the establishment of $\mathrm{CCl}$ (Fig. 5A,6A). The number of microglia expressed by P2X7R+ ${ }^{+}-1 \mathrm{ba} 1^{+}$in chronic pain rats was significantly higher than that in control rats $(39.18 \pm 2.28$ for group $V, 38.14 \pm 5.95$ for group $P, 79.37 \pm$ 8.35 for group $C, p<0.001,44.95 \pm 1.64$ for CP group, $p=0.001$, Fig. $5 B ; 3.42 \pm 0.28$ for group $V, 3.46 \pm 0.11$ for group $P, 8.61 \pm 0.81$ for group $C, P<0.001,6.96 \pm 1.01$ for CP group, $p<0.001$, Fig. $6 \mathrm{~B}$ ), moreover, $\mathrm{P} 2 \mathrm{X} 7 \mathrm{R}$ inhibitor treatment resulted in a decrease in the number of $\mathrm{P} 2 \mathrm{X} 7 \mathrm{R}^{+}-\mathrm{lba}{ }^{+}$microglia in the chronic pain group of rats (group $C$ vs. $C P, p<0.001$, Fig. 5B; group C vs. $C P, p=0.034$, Fig. $6 \mathrm{~B}$ ), suggesting that $\mathrm{P} 2 \mathrm{X} 7 \mathrm{R}$ in microglia mediates neuropathic pain.

However, we did not identify any P2X7R-positive cells that were also positive for the astrocytic marker GFAP in the samples, there was no significant difference between groups. (Fig. 5C, 6C) The variation trend of P2X7R was consistent with other experimental results. We further applied A-740003, the antagonist of P2X7R, by microinjection injection, and found it significantly decreased the content of $\mathrm{P} 2 \mathrm{X} 7 \mathrm{R}$ in the chronic group. This may confirm that P2X7R expressed in microglia plays a role in chronic pain, but P2X7R expressed in astrocytes does not.

\section{Microglia autophagy in MPFC and spinal cord contributed to chronic pain but not in astrocytes}

The results of a double P62 and microglial immunostaining of mPFC and spinal cord

(Fig. 7A, 8A) show that P62 was predominantly co-localized with microglia. The number of microglia expressed by $\mathrm{P} 62^{+}-\mathrm{Iba} 1^{+}$in chronic pain rats was significantly higher than that in control rats $(9.36 \pm 1.73$ for group $V, 9.47 \pm 3.12$ for group $P, 41.63 \pm 3.80$ for group $C, p<0.001,34.85 \pm 4.21$ for CP group, $P<$ 0.001 , Fig. 7B; $2.91 \pm 1.19$ for group $V, 3.16 \pm 0.38$ for group $P, 9.72 \pm 1.64$ for group $C, p<0.001,6.06 \pm$ 1.20 for CP group, $p<0.001$, Fig. 8B), moreover, P2X7R inhibitor treatment resulted in a decrease in the 
number of $\mathrm{P} 62^{+}-\mathrm{Iba} 1^{+}$microglia in the chronic pain group of rats (group $\mathrm{C}$ vs. $\mathrm{CP}, p=0.001$, Fig. 7B; group $C$ vs. $C P, p<0.001$, Fig. $8 B$ ), suggesting that autophagy in microglia mediates neuropathic pain. However, P62-positive cells that were also positive for the astrocytic marker GFAP in the samples (Fig. 7C, 8C), which was consistent with the trend of $\mathrm{P} 2 \mathrm{X} 7 \mathrm{R}^{+}-\mathrm{Iba} 1^{+}$cells. This also confirm that $\mathrm{P} 62$ expressed in microglia plays a role in chronic pain, but P62 expressed in astrocytes does not.

\section{Discussion}

It should be noted that neuropathic pain may cause the damage of tissue, central nerve fibers and cell, increase the probability of various neurodegenerative dysfunctions, such as chronic pain, sleep disorders, even mild cognitive impairment [16]. However, the mechanisms of neuropathic pain is unknown. The multiple neural circuits involved and the cause-and-effect mechanisms of chronic pain itself require further investigation.

In our experiment, we established $\mathrm{CCl}$, a widely used model of peripheral nerve injury. We have analyzed pain-related behaviors by PWT and PWL[16], consistent with previous studies[17], a stable hypersensitivity to pain after $\mathrm{CCl}$ establishment existed here, in addition, we have demonstrated that P2X7 inhibition rescues this CCl-treated phenotype, which verified that increased levels of P2X7 have participated in rats of $\mathrm{CCl}$. Previous results have shown that P2X7R plays a role in chronic pain [18], and changes in MPFC may be mediated through P2X7R in others experiments [19]. The dependence of the negative association between CCl-induced pain related behaviors and NP on the presence of P2X7 observed in this study suggests that P2X7 may be important and could function as a regulator of NP. Studies with animal models have suggested that neuropathic pain points to the structure and function of mPFC, which integrates the sensory, emotional and attentional components of pain perception, furthermore, spinal cord stimulation is reversible therapy for treatment of severe, otherwise nonresponsive chronic pain [20]. However, except for a few studies reporting the NP appeared after alterations of glutamatergic transmission in MPFC and spinal cord [21], little is known about the alterations in them and how they are affected. So this study was designed to observe changes in MPFC and spinal cord.

Additionally, this improved CCl-induced neuropathic pain in P2X7 inhibition animals, whether owing to an autophagy mechanism in mPFC and spinal cord, requires further proceeding. In the present study, we found that $\mathrm{CCl}$ increased P2X7 mRNA and protein expression in the mPFC and spinal cord of rat, demonstrating P2X7 may be an important participant in neuropathic pain. Recent studies have also demonstrated that high expression of P2X7R can be detected in MPFC and spinal dorsal horn cells. $\mathrm{P} 2 \mathrm{X7R}$ is closely related to neuronal activation, neurosensitization, pain transmission and neuroinflammation [22]. Moreover, autophagy also plays a key role in the pathological progression of pain. Studies have shown that the possible mechanism of the abnormal pain persisted after nerve injury is that the P2X7R mediated the sustained release of injury-related molecules, further promotes the formation of autophagy, when P2X7R impaired, the production of P2X7-triggered neuronal autophagy

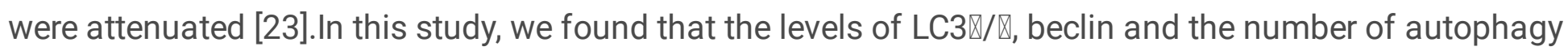
body in mPFC were significantly higher after after $\mathrm{CCl}$, and P2X7 inhibition showed an opposite condition. 
These results suggest that the autophagy activated by $\mathrm{CCl}$ was through $\mathrm{P} 2 \mathrm{X} 7$, the increased fluorescence under the electronic microscope also enhanced credibility [24]. Autophagy is a lysosomal degradation pathway and a homoeostatic cellular mechanism that is essential for survival, differentiation, development and homoeostasis. Meanwhile, recent investigations showed that autophagy was involved in neuropathic pain processing [25]. As a result of the dysfunction of autophagy, increased markers of autophagy (LC3区/ $\mathbb{Z}$ and Beclin) are observed in the early stages of $\mathrm{CCl}$, which represents the accumulation of dysfunctional autophagosomes. It has also been shown that $\mathrm{P} 2 \mathrm{X} 7$, as a positive regulator of the autophagic process, could apparently increase autophagy in glial. These demonstrated that the attenuated neuropathic pain after $\mathrm{CCl}$ in rat after $\mathrm{P} 2 \mathrm{X} 7$ inhibition are partly due to reductions in autophagy.

On a mechanistic ground, the CCl-induced autophagy has been demonstrated to involved modulation of apoptosis. In previous study, the accumulation of autophagy also could induced the increase of apoptosis [26, 27]. Adult apoptosis is a process that by which a cell ceases to grow and results in the controlled death of the cell [28]. Apoptosis is regulated by two large families of proteins, the caspase family and the Bcl-2 family. The ratio of $\mathrm{Bcl}-2 / \mathrm{Bax}$ is an indicator of cell susceptibility to apoptosis, with a decreasing ratio indicating increase in apoptosis [29]. The caspases are a family of protease enzymes. They are mainly classified into initiators such as caspase- 9 and effectors such as caspase- 3 . In order to know whether the decrease apoptosis may result from the change of the $\mathrm{Bcl}-2 / \mathrm{Bax}$ ratio and caspase-3, we quantified the level of them. As expected, the ratio of Bcl-2/Bax was reduced and caspase- 3 was significantly increased after $\mathrm{CCl}$. In addition, all of changes were less severe in P2X7 inhibition mice. A correlation has been shown between NP and decreased plasma levels of Bcl-2/Bax ratio [30]. Moreover, P2X7 activation and an increase in the autophagy can reduce adult apoptosis and produce the pain. It has been proven that P2X7R existed in neurons, and its over-expression can cause neuronal damage. It is also found, after tissue damage, that P2X7R was highly expressed in neurons, up-regulated the expression levels of caspase-3 and increased neuronal apoptosis [31]. Application of P2X7 inhibitor can inhibit neurons apoptosis and reduce tissue damage [32]. When P2X7 was activated by $\mathrm{CCl}$ in our study, it could increase cell membrane permeability, and the production of P2X7-triggered inflammatory factors and apoptosis were increased to activate microglia [32], which result in pain induced by tissue injury. These suggested that apoptosis activated by P2X7 was implicated in CCl-induced autophagy.

Numerous studies have predominantly demonstrated a critical role of glia in the development of pain hypersensitivity in neuropathic pain [33]. During the process of NP, microglial cells become hypertrophied and have increased IBA-1 expression; while astrocytes become hypertrophied with an increased expression of GFAP for instance. The activated glial cells have been reported was activate by P2X7R, and released other various cytokines to sustain plasticity changes in neurons and the eventual development of NP [34]. In our study, the microglial and astroglial were significantly activated after $\mathrm{CCl}$, which were reversed in P2X7 inhibition rats. After peripheral nerve injury, microglia cells activate and change their morphology, and neuropathic pain is caused by neuropathic active factors and cytokines released. But consistently with some previous studies [35], our results exhibited that P2X7R is predominantly localized to microglia throughout the CNS after CCI establishment, but not localized to astrocytes. The activation 
of microglial plays a key role in the development and transmission of pain. Huang et al. reported that P2X7R is expressed mostly in microglia, but not in astrocytes [36, 37]and P2X7R is upregulated along with microglia activation by the development of pain in rats [37], induce abnormal neuronal activity, enhance synaptic transmission of nociceptive neurons, and produce abnormal pain [38], and antagonism of P2X7R can significantly attenuated pain and reduced the upregulation of P2X7R as well as microglia activity in rats [39]. On the other hand, over-expression of P2X7R by studying animal models induced NP can activate microglial, increase the levels Tumor necrosis factor-alpha (TNF-a)and IL-1 $\beta$, and result in hyperalgesia and allodynia [40] [41]. These demonstrated that P2X7R expressed in microglia plays a crucial role in the development of NP.

Microglial autophagy is suggested to be involved in modulating homeostasis in the central nervous system, when it was activated, it could directly enhance the excitability of pain-transmitting neurons [42], and increased autophagy activity exerted a protective effect in NP via inflammasome inactivation [7]. Given the same trend of glial seen with neuropathic pain in the spinal cord vs. the MPFC, it is not surprising that our data showed P2X7R activation in microglia appears to play an important role in pain regulation in both the spinal cord and brain, through autophagy.

There are some limitations to our study. First, P2X7R inhibitor may represent a limitation because the half time of drugs. Second, we did not observe the damage effects of P2X7R on CCl-induced NP using agonist and we didn't verify our research through systematical injection. In addition, only the most basic measurements of the autopaghy and apoptosis have been researched, and the downstream mechanism has not been further investigated. Finally, mPFC and spinal cord were investigated, but we did not study which was in the upstream.

In this study, we found a pronounced increase of P2X7R expression in rats of $\mathrm{CCl}$, which confirming a possible correlation with CCl-induced NP. Conversely, P2X7R inhibition can counteract the CCl-induced NP due to autophagy and apoptosis via a modulation of microglia in mPFC and spinal cord. This may provide an importantly neuroprotective mechanism for the improved NP and also help devising new therapeutic to improve chronic pain in patients.

\section{Abbreviations}

NP

Neuropathic pain; mPFC:medial prefrontal cortex;IL-1 13 :Interleukin-1 $\beta$; CCI:Chronic-Constriction Injury; Veh:vehicle group; $\mathrm{C}$ group:CCl group; $\mathrm{P}$ group: $\mathrm{P} 2 \mathrm{X} 7$ inhibitor group; $\mathrm{CP}$ group: $\mathrm{CCl}+\mathrm{P} 2 \mathrm{X} 7 \mathrm{R}$ inhibition group; PWT:paw-withdrawal threshold; PWL:paw-withdrawal latency; BCA:bicinchoninic acid; ECL:enhanced chemiluminescence; TEM:transmission electron microscopy; IBA-1:ionized calcium binding adaptor molecule 1; GFAP:glial acidic fibrillary protein; SEM:standard error of the mean; ANOVA:one-way analysis of variance; TNF-a:Tumor necrosis factor-alpha

\section{Declarations}


Acknowledgements

This work was conducted at Zhongnan Hospital, Wuhan University. Meanwhile, thanks the native English speaking editors at American Journal Experts for language help.

\section{Authors' contributions}

QZ and YH performed most of the experiments and wrote the manuscript. CC and ZZ were responsible for the original design and providing key revisions, which are important for the content. YH completed behavioral Assays, data management and statistical analysis. TL,PF and JC participated in the experimental design and coordination. All authors contributed and approved the final version of the manuscript.

\section{Funding}

This work was supported by the National Natural Science Foundation of China (81771160), Natural Science Foundation of Hubei Province, China (No.2019CFB106), Cultivation fund from Zhongnan Hospital, Wuhan University (znpy2018092).

\section{Availability of data and materials}

The data supporting the findings of this study are included within the article.

\section{Ethics approval}

All animal protocols were reviewed and approved by the Animal Ethics Committee of the Zhongnan Hospital and Research Center in Hubei, China, and all animal experiments complied with the ARRIVE guidelines and was carried out in accordance with the National Institutes of Health guide for the care and use of Laboratory animals (NIH Publications No. 8023, revised 1978).

\section{Consent for publication}

Not applicable.

\section{Competing interests}

The authors declare that they have no competing interests

\section{References}

[1] Lin JP, Chen CQ, Huang LE, Li NN, Yang Y, Zhu SM, Yao YX. Dexmedetomidine Attenuates Neuropathic Pain by Inhibiting P2X7R Expression and ERK Phosphorylation in Rats. EXP NEUROBIOL 2018;27:267-76.

[2] Shohayeb B, Diab M, Ahmed M, Ng D. Factors that influence adult neurogenesis as potential therapy. TRANSL NEURODEGENER 2018;7:4. 
[3] Pinho-Ribeiro FA, Verri WJ, Chiu IM. Nociceptor Sensory Neuron-Immune Interactions in Pain and Inflammation. TRENDS IMMUNOL 2017;38:5-19.

[4] Cheriyan J, Sheets PL. Altered Excitability and Local Connectivity of mPFC-PAG Neurons in a Mouse Model of Neuropathic Pain. J NEUROSCI 2018;38:4829-39.

[5] Piao Y, Gwon DH, Kang DW, Hwang TW, Shin N, Kwon HH, Shin HJ, Yin Y, Kim JJ, Hong J, Kim HW, Kim Y, Kim SR, Oh SH, Kim DW. TLR4-mediated autophagic impairment contributes to neuropathic pain in chronic constriction injury mice. MOL BRAIN 2018;11:11.

[6] Groh A, Krieger P, Mease RA, Henderson L. Acute and Chronic Pain Processing in the Thalamocortical System of Humans and Animal Models. NEUROSCIENCE 2018;387:58-71.

[7] Chen H, Zhou C, Xie K, Meng X, Wang Y, Yu Y. Hydrogen-rich Saline Alleviated the Hyperpathia and Microglia Activation via Autophagy Mediated Inflammasome Inactivation in Neuropathic Pain Rats. NEUROSCIENCE 2019;421:17-30.

[8] Medeiros P, Negrini-Ferrari SE, Palazzo E, Maione S, Ferreira SH, de Freitas RL, Coimbra NC. Nmethyl-D-aspartate Receptors in the Prelimbic Cortex are Critical for the Maintenance of Neuropathic Pain. NEUROCHEM RES 2019;44:2068-80.

[9] Karmakar M, Katsnelson MA, Dubyak GR, Pearlman E. Neutrophil P2X7 receptors mediate NLRP3 inflammasome-dependent IL-1beta secretion in response to ATP. NAT COMMUN 2016;7:10555.

[10] Tsuda M. P2 receptors, microglial cytokines and chemokines, and neuropathic pain. J NEUROSCI RES 2017;95:1319-29.

[11] Kambur O, Kaunisto MA, Winsvold BS, Wilsgaard T, Stubhaug A, Zwart JA, Kalso E, Nielsen CS. Genetic variation in P2RX7 and pain tolerance. PAIN 2018;159:1064-73.

[12] Congdon EE, Sigurdsson EM. Tau-targeting therapies for Alzheimer disease. NAT REV NEUROL 2018;14:399-415.

[13] Guo F, Liu X, Cai H, Le W. Autophagy in neurodegenerative diseases: pathogenesis and therapy. BRAIN PATHOL 2018;28:3-13.

[14] Zhang M, Deng YN, Zhang JY, Liu J, Li YB, Su H, Qu QM. SIRT3 Protects Rotenone-induced Injury in SH-SY5Y Cells by Promoting Autophagy through the LKB1-AMPK-mTOR Pathway. AGING DIS 2018;9:273-86.

[15] Orioli E, De Marchi E, Giuliani AL, Adinolfi E. P2X7 Receptor Orchestrates Multiple Signalling Pathways Triggering Inflammation, Autophagy and Metabolic/Trophic Responses. CURR MED CHEM 2017;24:2261-75. 
[16] Chen H, Hu Y, Xie K, Chen Y, Wang H, Bian Y, Wang Y, Dong A, Yu Y. Effect of autophagy on allodynia, hyperalgesia and astrocyte activation in a rat model of neuropathic pain. INT J MOL MED 2018;42:2009-19.

[17] Weng W, Yao C, Poonit K, Zhou X, Sun C, Zhang F, Yan H. Metformin relieves neuropathic pain after spinal nerve ligation via autophagy flux stimulation. J CELL MOL MED 2019;23:1313-24.

[18] Fischer W, Franke H, Krugel U, Muller H, Dinkel K, Lord B, Letavic MA, Henshall DC, Engel T. Critical Evaluation of P2X7 Receptor Antagonists in Selected Seizure Models. PLOS ONE 2016;11:e156468.

[19] Inoue K. Purinergic signaling in microglia in the pathogenesis of neuropathic pain. Proc Jpn Acad Ser B Phys Biol Sci 2017;93:174-82.

[20] Woodroffe RW, Pearson AC, Pearlman AM, Howard MA, Nauta H, Nagel SJ, Hori YS, Machado AG, Almeida FL, Helland L, Holland MT, Gillies GT, Wilson S. Spinal Cord Stimulation for Visceral Pain: Present Approaches and Future Strategies. PAIN MED 2020;21:2298-309.

[21] Kelly CJ, Huang M, Meltzer H, Martina M. Reduced Glutamatergic Currents and Dendritic Branching of Layer 5 Pyramidal Cells Contribute to Medial Prefrontal Cortex Deactivation in a Rat Model of Neuropathic Pain. FRONT CELL NEUROSCI 2016;10:133.

[22] Lee DS, Kim JE. P2 $\times 7$ Receptor Inhibits Astroglial Autophagy via Regulating FAK- and PHLPP1/2-Mediated AKT-S473 Phosphorylation Following Kainic Acid-Induced Seizures. INT J MOL SCI 2020;21.

[23] Sekar P, Huang DY, Hsieh SL, Chang SF, Lin WW. AMPK-dependent and independent actions of $\mathrm{P} 2 \mathrm{X7}$ in regulation of mitochondrial and lysosomal functions in microglia. CELL COMMUN SIGNAL 2018;16:83.

[24] Jin GL, Yue RC, He SD, Hong LM, Xu Y, Yu CX. Koumine Decreases Astrocyte-Mediated Neuroinflammation and Enhances Autophagy, Contributing to Neuropathic Pain From Chronic Constriction Injury in Rats. FRONT PHARMACOL 2018;9:989.

[25] Zheng X, Chen L, Du X, Cai J, Yu S, Wang H, Xu G, Luo Z. Effects of hyperbaric factors on lidocaine-induced apoptosis in spinal neurons and the role of p38 mitogen-activated protein kinase in rats with diabetic neuropathic pain. EXP THER MED 2017;13:2855-61.

[26] Wang K. Autophagy and apoptosis in liver injury. CELL CYCLE 2015;14:1631-42.

[27] Linden J, Koch-Nolte F, Dahl G. Purine Release, Metabolism, and Signaling in the Inflammatory Response. ANNU REV IMMUNOL 2019;37:325-47. 
[28] Kun L, Lu L, Yongda L, Xingyue L, Guang H. Hyperbaric oxygen promotes mitophagy by activating CaMKKbeta/AMPK signal pathway in rats of neuropathic pain. MOL PAIN 2019;15:2069292139.

[29] Khodapasand E, Jafarzadeh N, Farrokhi F, Kamalidehghan B, Houshmand M. Is Bax/Bcl-2 ratio considered as a prognostic marker with age and tumor location in colorectal cancer? Iran Biomed J 2015;19:69-75.

[30] Hajializadeh Z, Nasri S, Kaeidi A, Sheibani V, Rasoulian B, Esmaeili-Mahani S. Inhibitory effect of Thymus caramanicus Jalas on hyperglycemia-induced apoptosis in in vitro and in vivo models of diabetic neuropathic pain. J ETHNOPHARMACOL 2014;153:596-603.

[31] Zhang WJ, Zhu ZM, Liu ZX. The role and pharmacological properties of the P2X7 receptor in neuropathic pain. BRAIN RES BULL 2020;155:19-28.

[32] Luo W, Feldman D, McCallister R, Brophy C, Cheung-Flynn J. P2X7R antagonism after subfailure overstretch injury of blood vessels reverses vasomotor dysfunction and prevents apoptosis. Purinergic Signal 2017;13:579-90.

[33] Han G, Liu K, Li L, Li X, Zhao P. The effects of hyperbaric oxygen therapy on neuropathic pain via mitophagy in microglia. MOL PAIN 2017;13:2071452658.

[34] Yang Y, Li H, Li TT, Luo H, Gu XY, Lu N, Ji RR, Zhang YQ. Delayed activation of spinal microglia contributes to the maintenance of bone cancer pain in female Wistar rats via P2X7 receptor and IL-18. J NEUROSCI 2015;35:7950-63.

[35] Yue N, Huang H, Zhu X, Han Q, Wang Y, Li B, Liu Q, Wu G, Zhang Y, Yu J. Activation of P2X7 receptor and NLRP3 inflammasome assembly in hippocampal glial cells mediates chronic stressinduced depressive-like behaviors. J Neuroinflammation 2017;14:102.

[36] Huang ZX, Lu ZJ, Ma WQ, Wu FX, Zhang YQ, Yu WF, Zhao ZQ. Involvement of RVM-expressed P2X7 receptor in bone cancer pain: mechanism of descending facilitation. PAIN 2014;155:783-91.

[37] Su WJ, Zhang T, Jiang CL, Wang W. Clemastine Alleviates Depressive-Like Behavior Through Reversing the Imbalance of Microglia-Related Pro-inflammatory State in Mouse Hippocampus. FRONT CELL NEUROSCI 2018;12:412.

[38] Yi Z, Ouyang S, Zhou C, Xie L, Fang Z, Yuan H, Yang J, Zou L, Jia T, Zhao S, Li L, Shi L, Gao Y, Li G, Liu S, Xu H, Xu C, Zhang C, Liang S. Andrographolide Inhibits Mechanical and Thermal Hyperalgesia in a Rat Model of HIV-Induced Neuropathic Pain. FRONT PHARMACOL 2018;9:593.

[39] Zhou J, Zhang X, Zhou Y, Wu B, Tan ZY. Up-regulation of P2X7 Receptors Contributes to Spinal Microglial Activation and the Development of Pain Induced by BmK-I. NEUROSCI BULL 2019;35:624-36. 
[40] Shen Y, Guan S, Ge H, Xiong W, He L, Liu L, Yin C, Liu H, Li G, Xu C, Xu H, Liu S, Li G, Liang S, Gao Y. Effects of palmatine on rats with comorbidity of diabetic neuropathic pain and depression. BRAIN RES BULL 2018;139:56-66.

[41] Liu C, Tao J, Wu H, Yang Y, Chen Q, Deng Z, Liu J, Xu C. Effects of LncRNA BC168687 siRNA on Diabetic Neuropathic Pain Mediated by P2X7 Receptor on SGCs in DRG of Rats. BIOMED RES INT 2017;2017:7831251.

[42] Yang G, Tan Q, Li Z, Liu K, Wu J, Ye W, Mei H, Yu H. The AMPK pathway triggers autophagy during CSF1-induced microglial activation and may be implicated in inducing neuropathic pain. $J$ NEUROIMMUNOL 2020;345:577261.

\section{Figures}

a

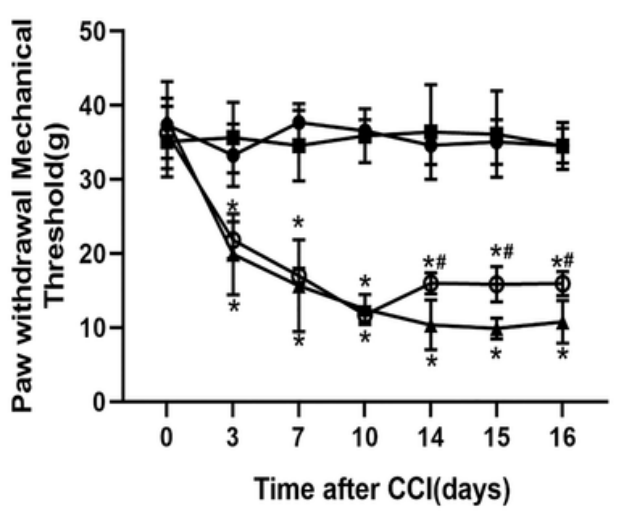

b

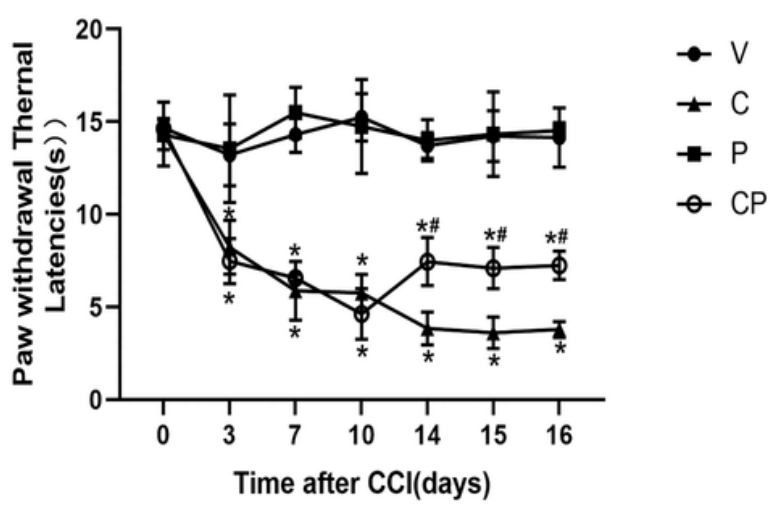

\section{Figure 1}

Inhibition of P2X7R can increase thermal and mechanical pain thresholds in rats with chronic neuropathic pain. All groups of rats were tested in paw-withdrawal threshold (PWT) (a) and pawwithdrawal latency (PWL) (b) after surgery ( $n=12$ /subgroup) within 3 consecutive days. The data were analyzed using two-way ANOVAs used Tukey's multiple comparisons test. Values are presented as the means \pm SD. Significant differences are expressed as follows: ${ }^{*} p<0.05$ vs. Veh group, $\# p<0.05$ vs. CP group for rats in $\mathrm{CCl}$ group. $\mathrm{C}=\mathrm{CCl}, \mathrm{P}=\mathrm{P} 2 \mathrm{X} 7 \mathrm{R}$ Inhibition, $\mathrm{CP}=\mathrm{CCI} \& \mathrm{P} 2 \mathrm{X7R}$ Inhibition. 
a

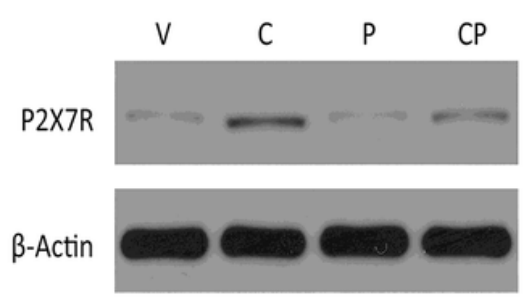

$69 \mathrm{kDa}$

$\beta$-Actin

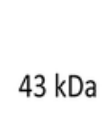

b

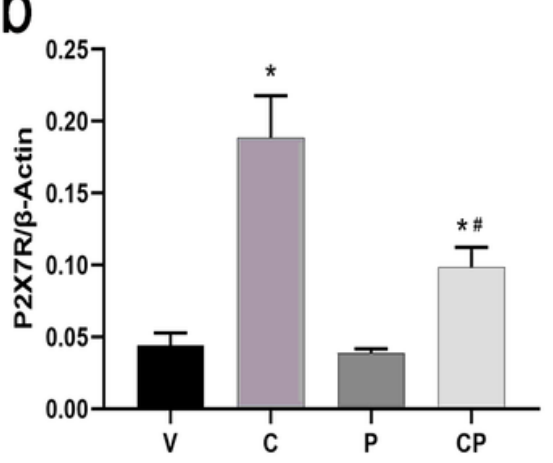

e

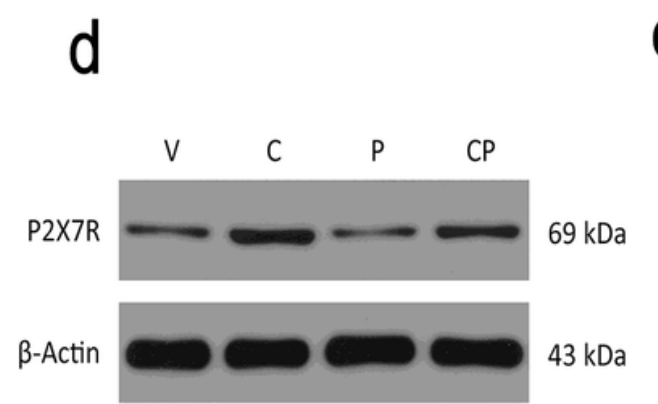

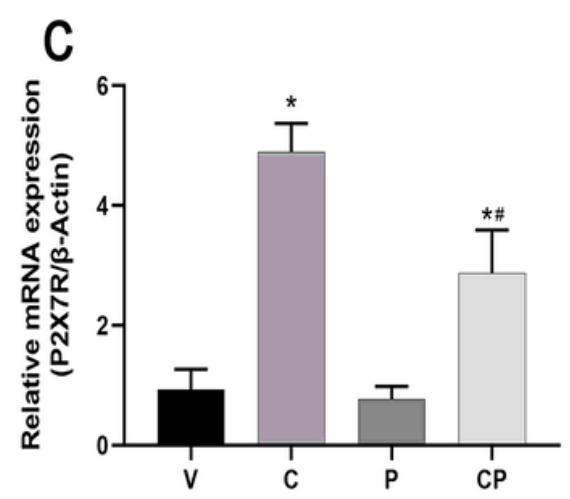

f

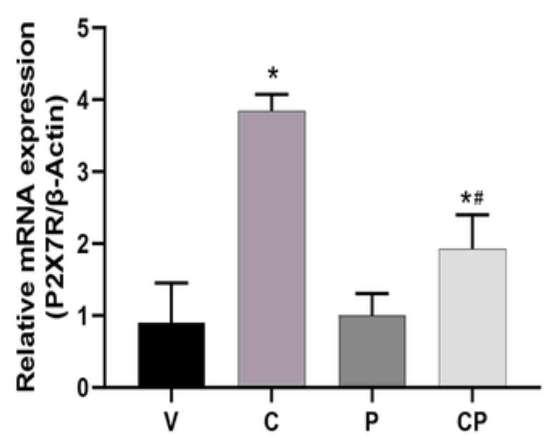

Figure 2

Chronic neuropathic pain was partially dependent on P2X7R in MPFC and spinal cord. The intensity of P2X7R in the mPFC $(a, b, c)$ and spinal cord $(d, e, f)$ of the rats were determined at 16 days after $\mathrm{CCl}$ surgery ( $n=4$ /subgroup). a,d. Western blotting of P2X7 protein from rats in the MPFC and spinal cord, respectively. b,e. $\beta$-actin was used as a loading control and the graphs represent the mean $\pm S D$ in Western blot. c,f. $\beta$-actin was used as a loading control and the graphs represent the mean \pm SD in PCR. The data were analyzed using two-way ANOVAs used Tukey's multiple comparisons test. Values are presented as the means \pm SD. Significant differences are expressed as follows: ${ }^{*}<<0.05$ vs. Veh group, $\# p<0.05$ vs. $\mathrm{CP}$ group for rats in $\mathrm{CCl}$ group. $\mathrm{C}=\mathrm{CCl}, \mathrm{P}=\mathrm{P} 2 \mathrm{X} 7 \mathrm{R}$ Inhibition, $\mathrm{CP}=\mathrm{CCl} \& \mathrm{P} 2 \mathrm{X} 7 \mathrm{R}$ Inhibition. 
a

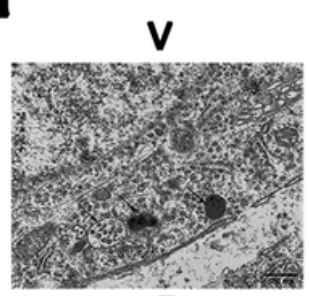

$\mathbf{P}$

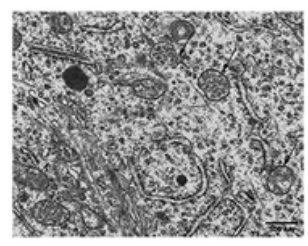

C
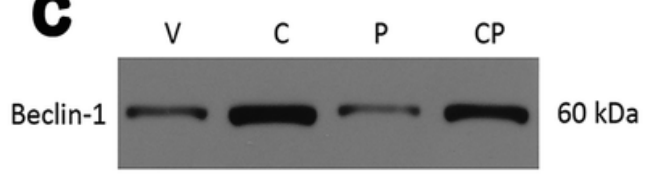

LC3

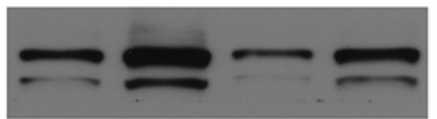

$16 \mathrm{kDa}$

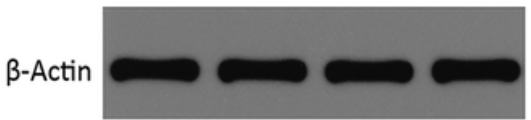

$43 \mathrm{kDa}$

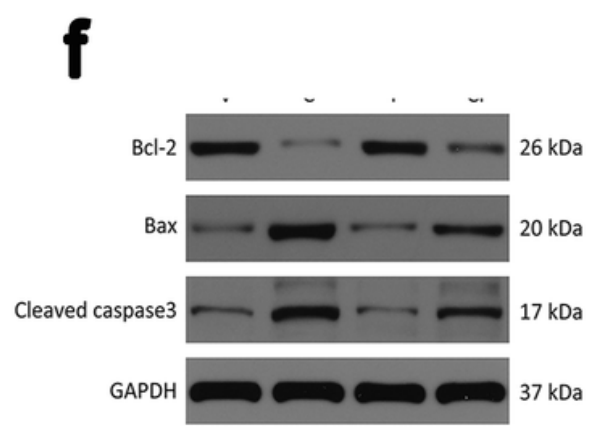

b

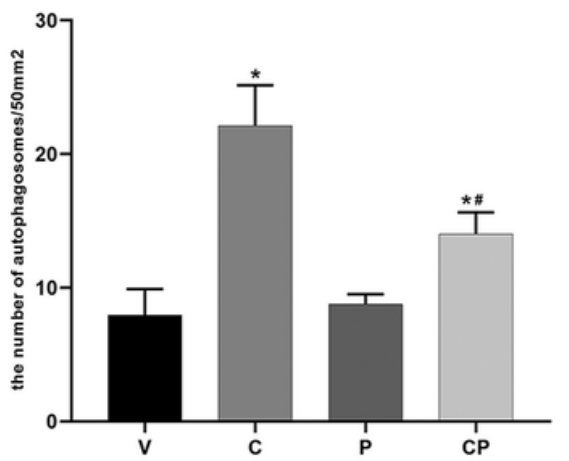

$\boldsymbol{\theta}$
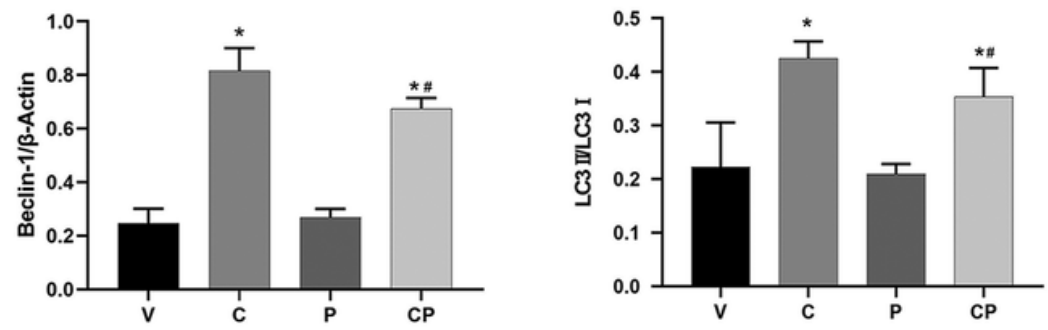

g

h

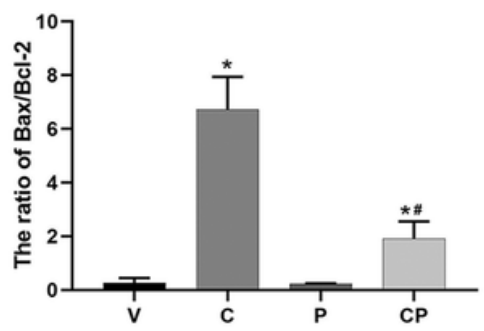

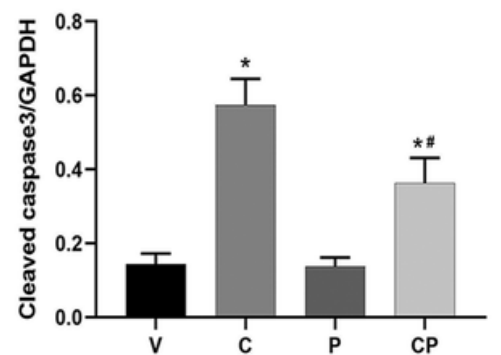

Figure 3

P2X7 elimination relieved chronic neuropathic pain through decreasing autophagy and apoptosis in $\mathrm{mPFC}$ of rats. a. Representative images of autophagosomes under transmission microscope in mPFC region of rats. Scale bar $=500 \mathrm{~nm}$. b. Quantitative analysis of the number of autophagosomes. c-e. Western blot analysis of autophagy -related proteins Beclin-1 and LC3, $\beta$-actin was used as an internal control. f-h. Western blot analysis of Bcl-2, Bax and cleaved-caspase- 3 in vivo experiment, $\beta$-actin was used as an internal control ( $n=4 /$ subgroup). Values are presented as the means \pm SD. Significant differences are expressed as follows: ${ }^{*} p<0.05$ vs. Veh group, $\# p<0.05$ vs. CP group for rats in CCI group. $\mathrm{C}=\mathrm{CCl}, \mathrm{P}=\mathrm{P} 2 \mathrm{X} 7 \mathrm{R}$ Inhibition, $\mathrm{CP}=\mathrm{CCl} \& \mathrm{P} 2 \mathrm{X7R}$ Inhibition. 
a

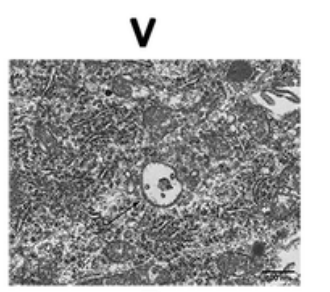

$\mathbf{P}$
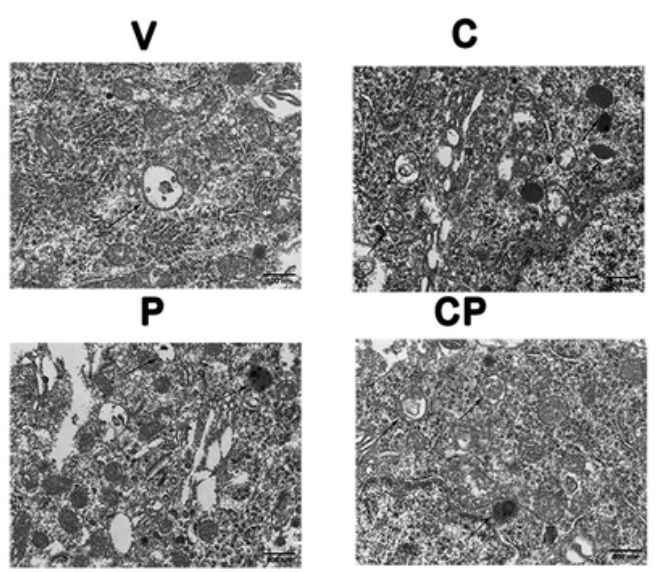

C

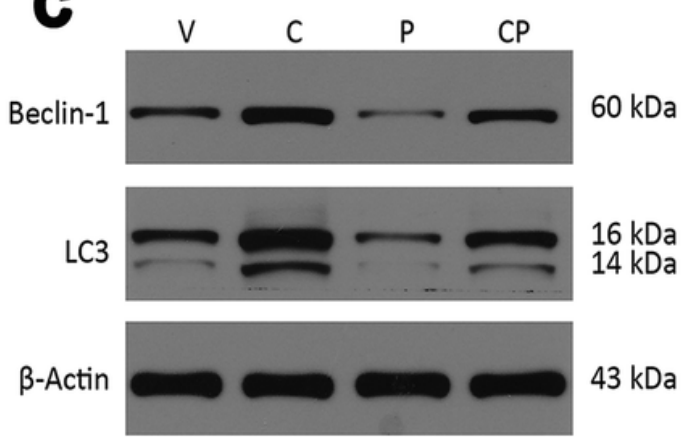

f

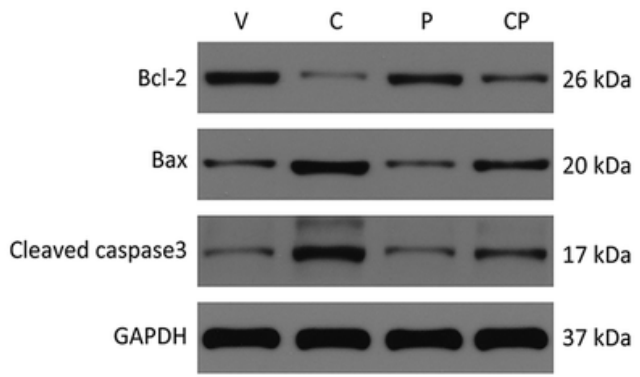

b

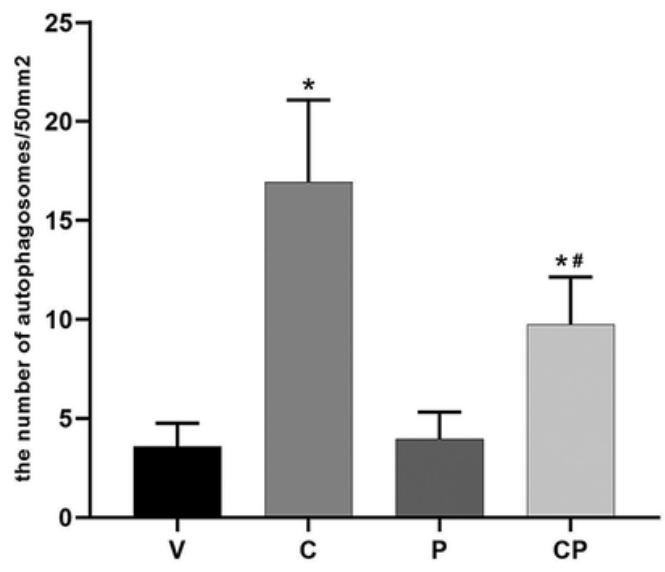

$\boldsymbol{\theta}$

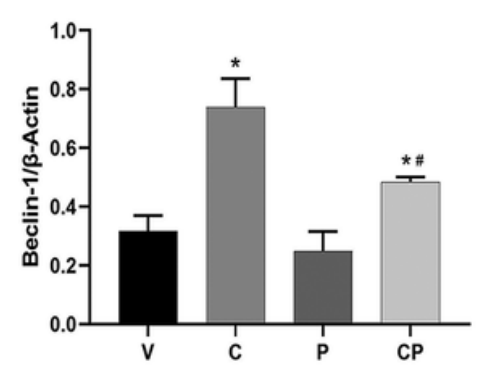

g

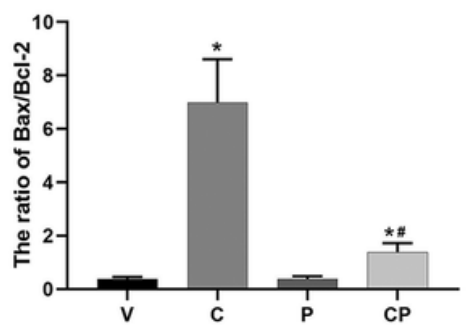

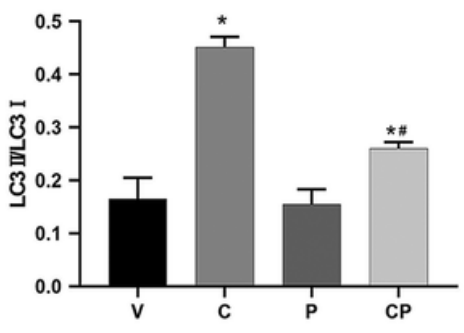

h

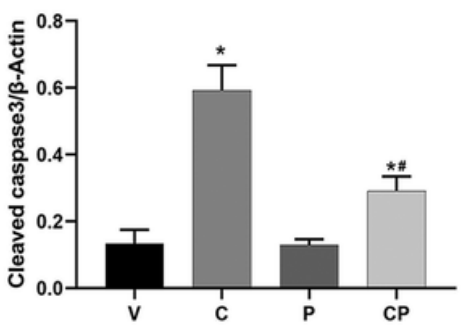

Figure 4

P2X7 elimination relieved chronic neuropathic pain through decreasing autophagy and apoptosis in spinal cord of rats. a. Representative images of autophagosomes under transmission microscope in spinal cord of rats. Scale bar $=500 \mathrm{~nm}$. b. Quantitative analysis of the number of autophagosomes. c-e. Western blot analysis of autophagy -related proteins Beclin-1 and LC3, $\beta$-actin was used as an internal control. f-h. Western blot analysis of Bcl-2, Bax and cleaved-caspase- 3 in vivo experiment, $\beta$-actin was used as an internal control ( $n=4 /$ subgroup). Values are presented as the means \pm SD. Significant differences are expressed as follows: ${ }^{*} p<0.05$ vs. Veh group, $\# p<0.05$ vs. CP group for rats in CCI group. $\mathrm{C}=\mathrm{CCl}, \mathrm{P}=\mathrm{P} 2 \mathrm{X} 7 \mathrm{R}$ Inhibition, $\mathrm{CP}=\mathrm{CCl} \& \mathrm{P} 2 \mathrm{X} 7 \mathrm{R}$ Inhibition. 

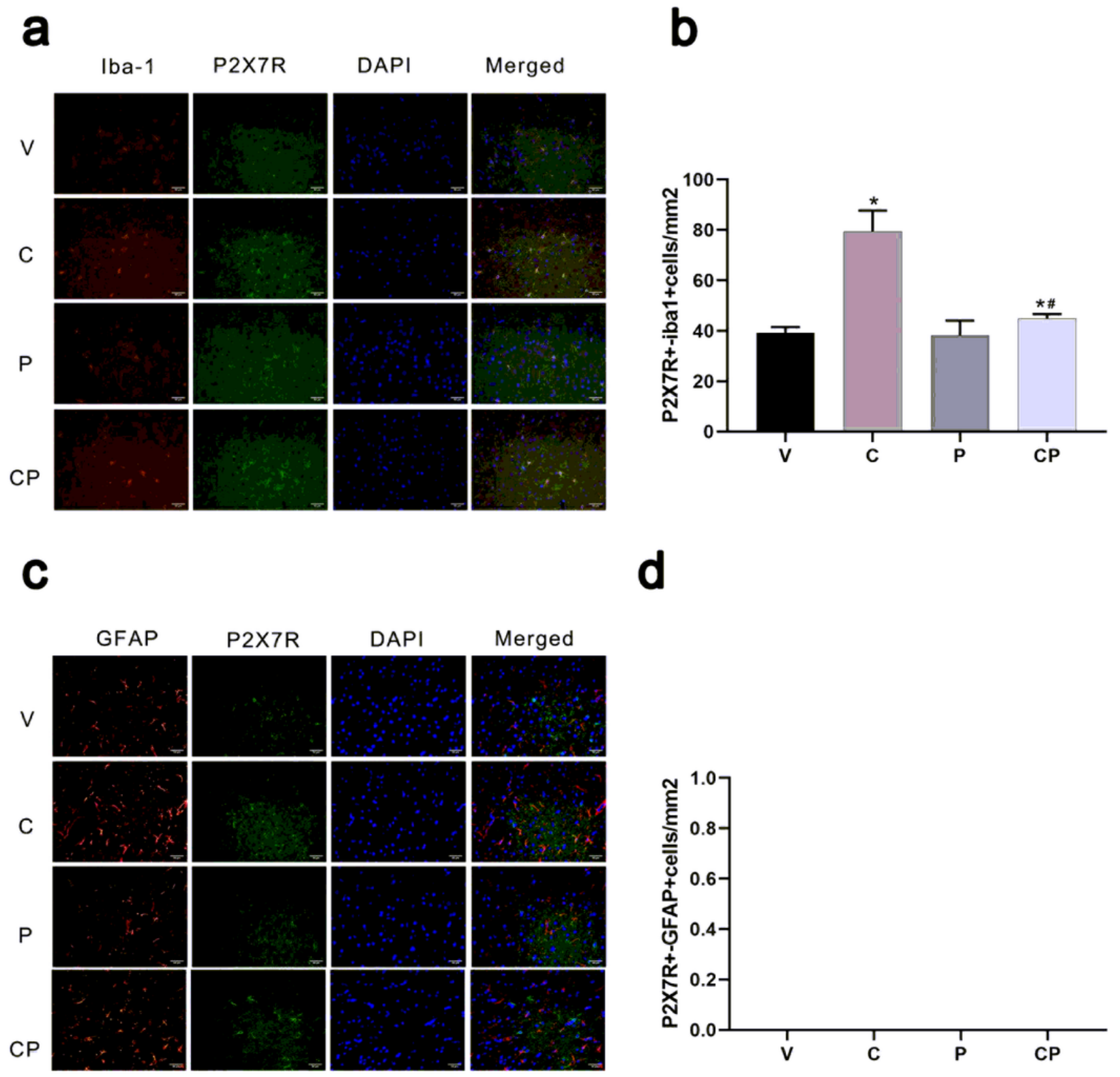

Figure 5

P2X7R in microglia of the brain contributed to chronic pain but not in astrocytes. P2X7R was predominantly co-localized with Iba1 (a microglial marker), but not with GFAP (astrocytic marker) in mPFC. a. Immunofluorescence staining of mPFC sections in microglial cells from rats. P2X7R (green), Iba1(red).b. Quantitative analysis of P2X7R+ and Iba1+ positive cells. c. Immunofluorescence staining of mPFC sections in microglial cells from rats. P2X7R (green), GFAP (red). d. Quantitative analysis of $\mathrm{P} 2 \mathrm{X7R}+$ and GFAP+ positive cells. Original magnification: $\times 100$; Scale bar $=50 \mu \mathrm{m}$. Values are presented 
as the means \pm SD. Significant differences are expressed as follows: * $p<0.05$ vs. Veh group, $\# p<0.05$ vs. $\mathrm{CP}$ group for rats in $\mathrm{CCl}$ group. $\mathrm{C}=\mathrm{CCl}, \mathrm{P}=\mathrm{P} 2 \mathrm{X} 7 \mathrm{R}$ Inhibition, $\mathrm{CP}=\mathrm{CCl} \& \mathrm{P} 2 \mathrm{X} 7 \mathrm{R}$ Inhibition.

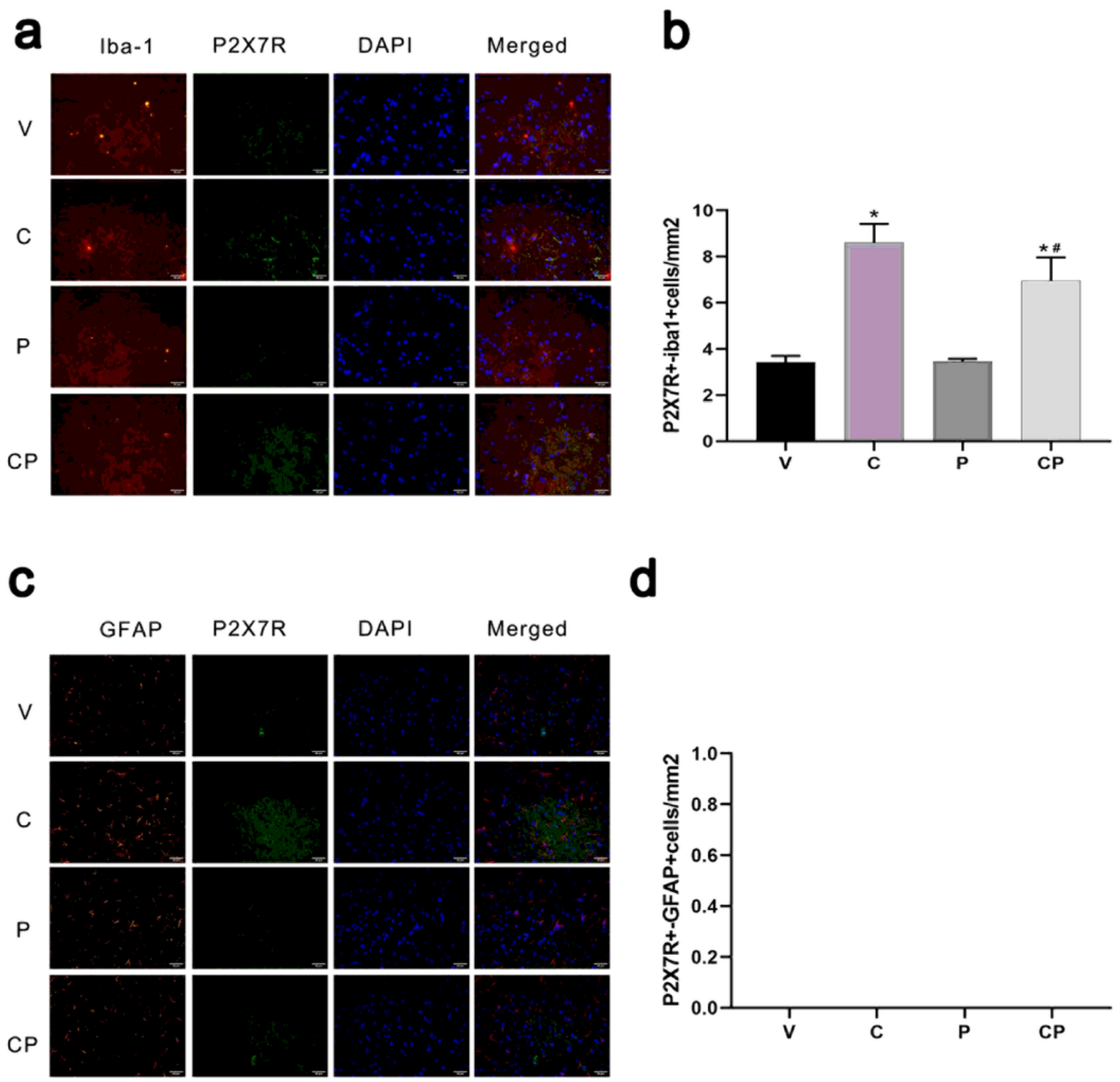

\section{Figure 6}

P2X7R in microglia of the spinal cord contributed to chronic pain but not in astrocytes P2X7R was predominantly co-localized with Iba1 (a microglial marker), but not with GFAP (astrocytic marker) in spinal cord. a. Immunofluorescence staining of spinal cord sections in microglial cells from rats. P2X7R (green), Iba1 (red). b. Quantitative analysis of P2X7R+ and Iba1+ positive cells. c. Immunofluorescence 
staining of spinal cord sections in microglial cells from rats. P2X7R (green), GFAP (red). d. Quantitative analysis of P2X7R+ and GFAP+ positive cells. Original magnification: $\times 100 ;$ Scale bar $=50 \mu \mathrm{m}$. Values are presented as the means \pm SD. Significant differences are expressed as follows: ${ }^{*} p<0.05 \mathrm{vs}$. Veh group, $\# \mathrm{p}<0.05$ vs. $\mathrm{CP}$ group for rats in $\mathrm{CCl}$ group. $\mathrm{C}=\mathrm{CCl}, \mathrm{P}=\mathrm{P} 2 \mathrm{X7R}$ Inhibition, $\mathrm{CP}=\mathrm{CCl} \& \mathrm{P} 2 \mathrm{X} 7 \mathrm{R}$ Inhibition.

a
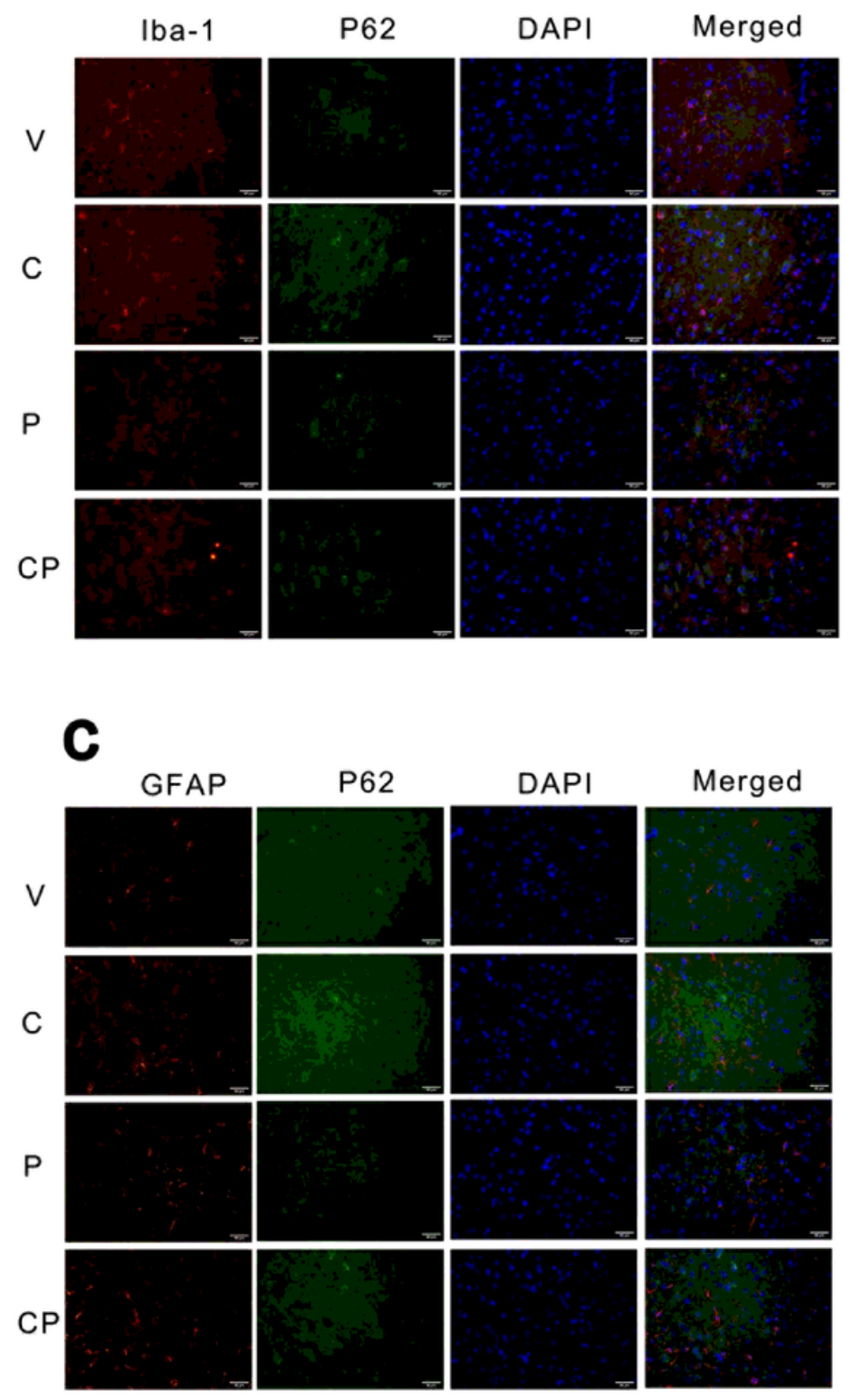

b
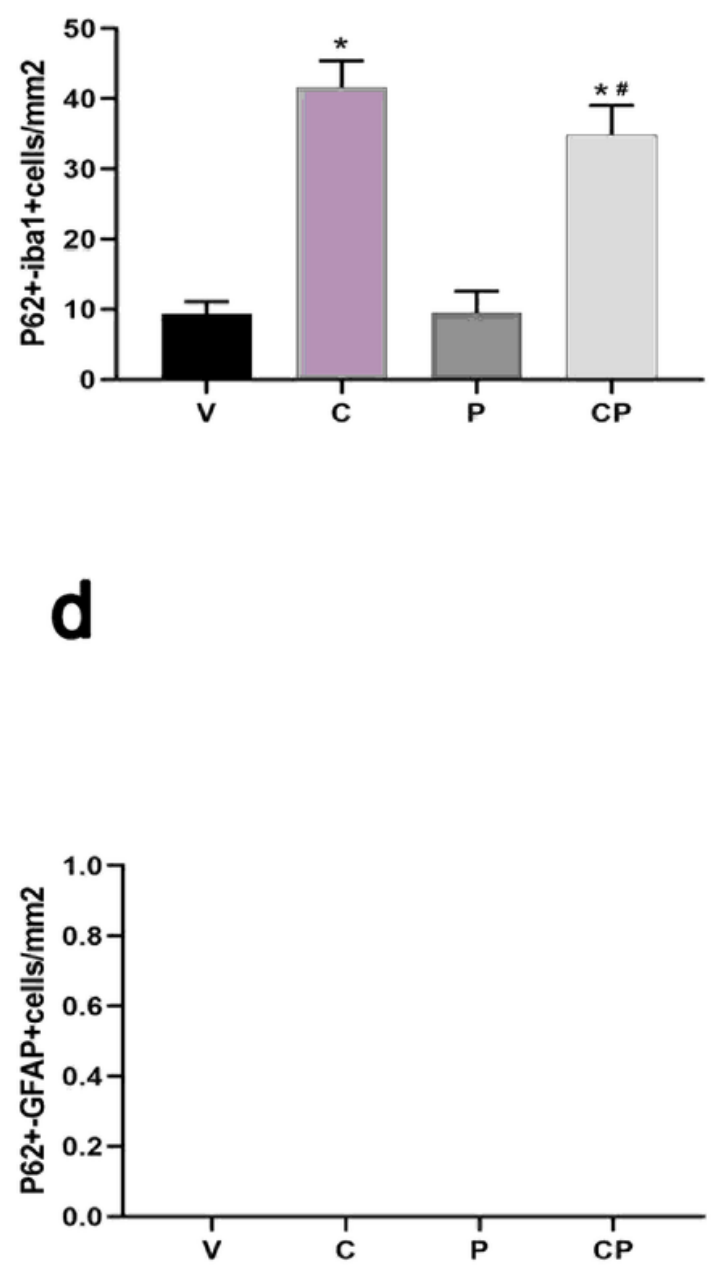

Figure 7 
Microglia autophagy in mPFC contributed to chronic pain but not in astrocytes. P62 was predominantly co-localized with Iba1 (a microglial marker), but not with GFAP (astrocytic marker) in mPFC. a.

Immunofluorescence staining of mPFC sections in microglial cells from rats. P62 (green), Iba1 (red). b. Quantitative analysis of P62+ and Iba1+ positive cells. c. Immunofluorescence staining of mPFC sections in microglial cells from rats. P62 (green), GFAP (red). d. Quantitative analysis of P62+ and GFAP+ positive cells. Original magnification: $\times 100$; Scale bar $=50 \mu \mathrm{m}$. Values are presented as the means \pm SD.

Significant differences are expressed as follows: ${ }^{*} p<0.05$ vs. Veh group, $\# p<0.05$ vs. CP group for rats in $\mathrm{CCl}$ group. $\mathrm{C}=\mathrm{CCl}, \mathrm{P}=\mathrm{P} 2 \mathrm{X} 7 \mathrm{R}$ Inhibition, $\mathrm{CP}=\mathrm{CCl} \& \mathrm{P} 2 \mathrm{X} 7 \mathrm{R}$ Inhibition.

\section{Supplementary Files}

This is a list of supplementary files associated with this preprint. Click to download.

- Additionalfile1.pdf 